\title{
Decidable Discriminator Varieties from Unary Varieties
}

\author{
Stanley Burris* Ralph McKenzie ${ }^{\dagger} \quad$ Matthew Valeriote ${ }^{\ddagger}$
}

\begin{abstract}
We determine precisely those locally finite varieties of unary algebras of finite type which, when augmented by a ternary discriminator, generate a variety with a decidable theory.
\end{abstract}

\section{Introduction}

Recent work of McKenzie and Valeriote [14] shows that if a locally finite variety has a decidable theory then it must decompose as the product of a strongly Abelian variety, an affine variety, and a discriminator variety. So, in order to completely characterize the decidable locally finite varieties of finite type, one must understand the structure of these three kinds of varieties, under the assumption of decidability.

In his PhD dissertation Valeriote [19] determined precisely when a locally finite strongly Abelian variety of finite type has a decidable theory. Prior to this, Burris and McKenzie [2] had reduced the decidability question for affine varieties to studying the decidability of the theory of all $\mathbf{R}$-modules, where $\mathbf{R}$ is any finite ring.

Thus the determination of all locally finite varieties with a decidable theory rests on determining those finite rings $\mathbf{R}$ such that the class of $\mathbf{R}$-modules has a decidable theory, and on determining those locally finite discriminator

\footnotetext{
*Research supported by NSERC Grant No. A7256

${ }^{\dagger}$ Research supported by the NSF

${ }^{\ddagger}$ Research supported by NSERC Grant No. URF0035169
} 
varieties which have a decidable theory. Both of these questions look very difficult at this point.

A variety $\mathcal{V}$ is a class of models of some algebraic language that is defined by a set of equations. Equivalently, a variety is a class of similar algebras closed under direct products, subalgebras and homomorphic images. The variety generated by a class $\mathcal{K}$ is the smallest variety which contains $\mathcal{K}$. It is denoted by $\mathrm{V}(\mathcal{K})$ and can be obtained by closing $\mathcal{K}$ under direct products, subalgebras and then homomorphic images.

Informally, a variety $\mathcal{V}$ in the language $L$ is said to be decidable if there is an algorithm to determine precisely which sentences of $L$ are true in every algebra in the variety. For a precise definition consult [3] or [14]. A variety is hereditarily undecidable if every subtheory of it is undecidable. For an explanation of the technique of semantic embedding that we use in this paper to establish our decidability results the reader may also consult [3] or $[14]$.

Discriminator varieties can be regarded as generalizations of the variety of Boolean algebras. On any set $U$ we can define an operation $t_{U}(x, y, z)$ by stipulating that $t_{U}(x, y, z)$ is $z$ if $x=y$, and is $x$ if $x \neq y$. This operation $t_{U}$ is called the ternary discriminator on $U$.

Definition 1.1 A variety $\mathcal{V}$ is called a discriminator variety iff there exists a term $t(x, y, z)$ in the language of $\mathcal{V}$ such that $\mathcal{V}=\mathrm{V}(\mathcal{S})$ where $\mathcal{S}$ is the class of all $\mathbf{A} \in \mathcal{V}$ such that $t^{\mathbf{A}}=t_{A}$ (i.e., the term $t$ defines the discriminator on the universe of $\mathbf{A}$ ). Such a term $t$ is called a discriminator term for $\mathcal{V}$.

Since every operation on the set $\{0,1\}$ is realized as a term operation of the two element Boolean algebra on this set, and since this algebra generates the variety of all Boolean algebras then it follows that the variety of all Boolean algebras is a discriminator variety. Other examples are listed below.

Definition 1.2 A Boolean product of an indexed family $\left(\mathbf{A}_{x}\right)_{x \in X}, X \neq \emptyset$, of algebras is a subdirect product $\mathbf{A} \leq \prod_{x \in X} \mathbf{A}_{x}$, where $X$ can be endowed with a Boolean space topology so that

(1) for $a, b \in A$, the set $\left\{x \in X: a_{x}=b_{x}\right\}$ is closed and open in $X$, and 
(2) if $a, b \in A$ and $N$ is a closed and open subset of $X$, then

$$
\left.\left.a\right|_{N} \cup b\right|_{X \backslash N} \in A .
$$

Condition (2) is referred to as the 'patchwork property'. For a class $\mathcal{K}$, $\Gamma^{a}(\mathcal{K})$ will denote the class of all Boolean products which can be formed from subsets of $\mathcal{K}$.

The following theorem summarizes some of the important properties of discriminator varieties. The proof of this theorem can be found in [3]

THEOREM 1.3 Let $\mathcal{K}$ be a class of algebras and let $t(x, y, z)$ be a discriminator term for $\mathcal{K}$. Then

(i) The simple algebras of $\mathrm{V}(\mathcal{K})$ are precisely the members of $\operatorname{ISP}_{\mathrm{U}}\left(\mathcal{K}_{+}\right)$, where $\mathcal{K}_{+}$is $\mathcal{K}$, augmented by a one-element algebra.

(ii) Every member of $\mathrm{V}(\mathcal{K})$ is isomorphic to a Boolean product of simple algebras, i.e., $\mathrm{V}(\mathcal{K})=\mathrm{I}^{a}{ }^{a} \mathrm{SP}_{\mathrm{U}}\left(\mathcal{K}_{+}\right)$.

It follows from the above theorem, that every discriminator variety is term equivalent to a variety of the form $\mathrm{V}\left(\mathcal{K}^{t}\right)$, where $\mathcal{K}$ is a class of algebras (in some language $\mathrm{L}$ ) defined by a set of universal sentences, and $\mathcal{K}^{t}$ is the class obtained by adjoining a new ternary function symbol $t$ to $L$ and interpreting it as the discriminator operation in all members of $\mathcal{K}$. It also follows that if $\mathcal{K}$ has an undecidable theory, then so does $\mathrm{V}\left(\mathcal{K}^{t}\right)$. Thus, in order to study the decidability question for locally finite discriminator varieties, it suffices to examine varieties of the form $\mathrm{V}\left(\mathcal{K}^{t}\right)$, where $\mathcal{K}$ is a decidable, locally finite universal class of algebras.

Although not needed in this paper, it is true that if $\mathcal{K}$ is a locally finite universal class of algebras in a finite language, then $\mathrm{V}\left(\mathcal{K}^{t}\right)$ is also locally finite. In fact, $\operatorname{HSP}(\mathcal{K})$ is a locally finite variety. An elementary argument using the Compactness Theorem proves this.

Let us quickly review the literature dealing with the decidability of discriminator varieties. The inspiring original source for all of the positive results was the decidability of the theory of Boolean algebras due to Tarski [16]. This was extended by Ershov [7] to the variety of relatively complemented distributive lattices, and in another direction he generalized this to Post varieties [8]. The real thrust of the recent work began with Comer's proof 
[5] that the variety of $x^{m} \approx x$-rings is decidable. He developed a version of the Feferman-Vaught theorem for certain Boolean sheaves, a technique which is used in this paper as well. Next Comer [6] showed that every proper subvariety of the variety of monadic algebras (a locally finite discriminator variety) has a decidable theory, and then Rubin [15] proved that the variety of all monadic algebras has an undecidable theory. Comer's techniques were extended in Burris and Werner[4] to encompass all finitely generated discriminator varieties. McKenzie [12] proved that the pure discriminator variety $\left[=\mathrm{V}\left(\omega^{t}\right)\right]$ has a decidable theory. In [1] Burris proved that nontrivial iterated discriminator varieties have undecidable theories i.e., for any discriminator variety $\mathcal{D}$, the variety $\mathrm{V}\left(\mathcal{D}^{t}\right)$ is undecidable.

In this paper we add to our very limited understanding of locally finite discriminator varieties by determining precisely those locally finite strongly Abelian varieties $\mathcal{K}$ of finite type such that the discriminator variety $\mathrm{V}\left(\mathcal{K}^{t}\right)$ is decidable. For now it will suffice to note that every unary algebra is strongly Abelian. We will have more to say of strongly Abelian algebras in section 4 .

\section{Undecidability}

Although rather technical, the following lemma has turned out to be very useful in establishing the undecidability of certain discriminator varieties.

LEMMA 2.1 Let A be an L-algebra with an infinite subset M. Let $\mathbf{S}$ be the subalgebra of A generated by $M$. Suppose the following holds:

(i) In A there is a first order definable relation, Equiv $(x, y)$, whose restriction to $S$ defines an equivalence relation $\equiv$ such that

a) no 2 elements from $M$ are related under $\equiv$,

$\mathbf{b )} \equiv$ is invariant under automorphisms of $\mathbf{S}$.

(ii) any bijection between two finite subsets of $M$ extends to an automorphism of $\mathbf{S}$.

(iii) for any $J \subseteq M$ finite, the only elements from $M$ that are $\equiv$-related to an element from the subalgebra of $\mathbf{A}$ generated by $J$ belong to $J$. 
(iv) there is a first order formula, $\mu(x)$, such that for $a \in S, \mathbf{A} \models \mu(a)$ if and only if $a \equiv m$ for some $m \in M$. Note that this implies that the subset of $A$ defined by $\mu$ is closed under $\equiv$.

(v) there is a first order formula, $\tau(x)$, such that

a) $\mathbf{A} \models \tau(a)$ for at least two elements from $M$,

b) $\mathbf{A} \models \neg \tau(b)$ for $\kappa$ many elements from $M$, for some infinite cardinal $\kappa$,

c) the subset of $A$ defined by $\tau$ is closed under $\equiv$.

Then the class of all graphs of size at most $\kappa$ can be semantically embedded into the class $\mathrm{P}_{\mathrm{s}}\left(\mathbf{A}^{t}\right)$, and hence the latter class is hereditarily undecidable.

Proof. Let $\mathbf{G}=\langle V, R\rangle$ be a graph with $|V| \leq \kappa$. By a graph we mean a set equipped with a binary relation that is symmetric and irreflexive. By an edge of the graph $\mathbf{G}$ we mean an unordered pair $\{a, b\}$ from $V$ such that $\langle a, b\rangle \in R$. Without loss of generality we can assume that each vertex of $\mathbf{G}$ is in at least two edges. This is because one can easily semantically embed the class of all graphs into the class of graphs satisfying the property that every vertex is in at least two edges. Let $I$ be an index set of size $\kappa$. Let $\lambda: I \longrightarrow E(\mathbf{G})$, where $E(\mathbf{G})$ is the set of edges of $\mathbf{G}$, be such that $\lambda^{-1}(e)$ is infinite for $e \in E(\mathbf{G})$. For each $v \in V$ choose $f_{v} \in M^{I}$ such that for $i \in I$ and $u, v \in V$ we have

(1) $u \neq v \Rightarrow f_{u}(i) \neq f_{v}(i)$

(2) $f_{v}(i) \in \tau^{\mathbf{A}} \Leftrightarrow v \in \lambda(i)$.

Let $\mathbf{B}$ be the subalgebra of $\mathbf{A}^{I}$ generated by the set $\left\{f_{v}: v \in V\right\}$.

For $R\left(x_{1}, \ldots, x_{k}\right)$ a relation on the set $A$, and $\bar{g}$ a $k$-tuple of elements from $A^{I}$, we write $\llbracket R(\bar{g}) \rrbracket$ to denote the set $\left\{i: i \in I\right.$ and $\left.R\left(g_{1}(i), \ldots, g_{k}(i)\right)\right\}$.

Claim 2.2 For $g, h \in B$ we have $\llbracket g=h \rrbracket, \llbracket g \equiv h \rrbracket \in\{\emptyset, I\}$.

Proof. Choose terms $p\left(x_{1}, \ldots, x_{n}\right)$ and $q\left(x_{1}, \ldots, x_{n}\right)$ and vertices $v_{1}, \ldots, v_{n}$ such that

$$
g=p^{\mathbf{B}}\left(f_{v_{1}}, \ldots, f_{v_{n}}\right)
$$


and

$$
h=q^{\mathbf{B}}\left(f_{v_{1}}, \ldots, f_{v_{n}}\right) .
$$

Let $i, j$ belong to $I$. Then by property (1) we have a bijection $\alpha$ from $\left\{f_{v_{1}}(i), \ldots, f_{v_{n}}(i)\right\}$ to $\left\{f_{v_{1}}(j), \ldots, f_{v_{n}}(j)\right\}$ defined by $\alpha\left(f_{v_{k}}(i)\right)=f_{v_{k}}(j)$, for $1 \leq k \leq n$ and by (ii) $\alpha$ lifts to an automorphism $\beta$ of $\mathbf{S}$.

If $i \in \llbracket g \neq h \rrbracket$, then this implies

$$
\begin{aligned}
& p^{\mathbf{S}}\left(f_{v_{1}}(i), \ldots, f_{v_{n}}(i)\right) \neq q^{\mathbf{S}}\left(f_{v_{1}}(i), \ldots, f_{v_{n}}(i)\right) \\
\Rightarrow & \beta p^{\mathbf{S}}\left(f_{v_{1}}(i), \ldots, f_{v_{n}}(i)\right) \neq \beta q^{\mathbf{S}}\left(f_{v_{1}}(i), \ldots, f_{v_{n}}(i)\right) \\
\Rightarrow & p^{\mathbf{S}}\left(f_{v_{1}}(j), \ldots, f_{v_{n}}(j)\right) \neq q^{\mathbf{S}}\left(f_{v_{1}}(j), \ldots, f_{v_{n}}(j)\right) \\
\Rightarrow & g(j) \neq h(j),
\end{aligned}
$$

and so $j \in \llbracket g \neq h \rrbracket$ too. From this it follows that $\llbracket g \neq h \rrbracket \in\{\emptyset, I\}$.

Now, suppose that $i \in \llbracket g \not \equiv h \rrbracket$. Then by replacing the inequalities in the above display by $\not \equiv$ 's and using properties (1) and (i) it follows that $j \in \llbracket g \not \equiv h \rrbracket$. Thus $\llbracket g \not \equiv h \rrbracket=\emptyset$ or $I$.

Let $C$ be the subset of $A^{I}$ consisting of those elements which are almost equal to a member of $B$. Then $C$ is a subuniverse of $\mathbf{A}^{I}$ such that

(3) $\{\llbracket g \neq h \rrbracket: g, h \in C\}$ is the collection of finite and cofinite subsets of $I$, and

(4) $C$ is closed under patchwork over the finite and cofinite subsets of $I$.

Property (3) follows immediately from the previous claim and property (4) follows from the definition of $C$. Thus $C$ is a subuniverse of $\left(\mathbf{A}^{t}\right)^{I}$, and the corresponding subalgebra $\mathbf{C}(t) \leq\left(\mathbf{A}^{t}\right)^{I}$ is actually a subdirect power of $\mathbf{A}^{t}$.

Claim 2.3 There is an effective procedure to define, for any first-order $\mathrm{L}(t)$ formula $\phi(\bar{x})$, a first-order $\mathrm{L}(t)$-formula $\phi^{*}(\bar{x})$ such that for $\bar{g} \in C$ we have

$$
\llbracket \phi^{\mathbf{A}^{t}}(\bar{g}) \rrbracket=I \Leftrightarrow \mathbf{C}(t) \models \phi^{*}(\bar{g}) .
$$

Proof. First note that in $\mathbf{C}(t)$ we have

$$
\llbracket f \neq g \rrbracket \subseteq \llbracket h \neq k \rrbracket \Leftrightarrow s(h, k, f, g)=g,
$$

where $s(x, y, u, v)$ is the term $t(t(x, y, u), t(x, y, v), v)$. Using this we can find a first-order $\mathrm{L}(t)$-formula atom $(x, y)$ such that 


$$
\llbracket f \neq g \rrbracket \text { is a singleton } \Leftrightarrow \mathbf{C}(t) \models \operatorname{atom}(f, g) .
$$

In view of (3) we have

$$
\{\{i\}: i \in I\}=\{\llbracket f \neq g \rrbracket: \mathbf{C}(t) \models \operatorname{atom}(f, g)\} .
$$

We assume that $\phi\left(x_{1}, \ldots, x_{n}\right)$ is in prenex form, say

$$
Q_{1} y_{1} \cdots Q_{m} y_{m} \omega\left(x_{1}, \ldots, x_{n}, y_{1}, \ldots, y_{m}\right),
$$

where $\omega$ is quantifier free and the $Q_{i}$ are either universal or existential quantifiers. Using the standard properties of $t$ and $s$, namely that $\mathbf{A}^{t}$ satisfies

$$
\begin{aligned}
& \left(z_{1} \approx w_{1} \vee z_{2} \approx w_{2}\right) \leftrightarrow s\left(z_{1}, w_{1}, z_{2}, w_{2}\right) \approx z_{2} \\
& \left(z_{1} \not w_{1} \vee z_{2} \approx w_{2}\right) \leftrightarrow s\left(z_{1}, w_{1}, z_{2}, w_{2}\right) \approx w_{2} \\
& \left(z_{1} \not \approx w_{1} \vee z_{2} \not \approx w_{2}\right) \leftrightarrow t\left(z_{1}, w_{1}, z_{2}\right) \not \approx t\left(w_{1}, z_{1}, w_{2}\right)
\end{aligned}
$$

we can find $\mathrm{L}(t)$-terms $p^{\prime}, q^{\prime}$ such that $\mathbf{A}^{t}$ satisfies $\omega \leftrightarrow p^{\prime} \approx q^{\prime}$ or $\mathbf{A}^{t}$ satisfies $\omega \leftrightarrow p^{\prime} \not q^{\prime}$. If the latter is the case choose two variables $x, y$ not occurring in $\phi$, and observe that $\mathbf{A}^{t}$ satisfies

$$
p^{\prime} \not \approx q^{\prime} \leftrightarrow \forall x \forall y\left[s\left(p^{\prime}, q^{\prime}, x, y\right) \approx y\right] .
$$

Thus we have a formula $\phi^{\prime}$ such that $\mathbf{A}^{t} \models \phi \leftrightarrow \phi^{\prime}$, and the matrix of $\phi^{\prime}$ is an atomic formula, say $p \approx q$. [This reduction was used by Burris and Werner [4] in the study of model companions. McKenzie [11] had earlier proved striking results about the spectra of an equation using a similar reduction of a first-order sentence to an equation $\phi^{*}$ in an expanded language.] Clearly $\llbracket \phi\left(g_{1}, \ldots, g_{n}\right) \rrbracket=I$ iff $\llbracket \phi^{\prime}\left(g_{1}, \ldots, g_{n}\right) \rrbracket=I$. Now, to define $\phi^{*}$ choose two variables $x, y$ not appearing in $\phi^{\prime}$ and replace $p \approx q$ in $\phi^{\prime}$ by $s(x, y, p, q) \approx p$ to get $\phi^{\prime \prime}$; then $\phi^{*}$ is $\forall x \forall y\left[\operatorname{atom}(x, y) \rightarrow \phi^{\prime \prime}\right]$.

Define $\Delta(x)$ to be $\mu^{*}(x)$. In the following we use $f \stackrel{\text { ae }}{=} g$ as an abbreviation for ' $\llbracket f \neq g \rrbracket$ is finite' and $f \stackrel{\text { ae }}{=} g$ as an abbreviation for ' $\llbracket f \not \equiv g \rrbracket$ is finite'.

Claim 2.4 (a) $\mathbf{C}(t) \models \Delta\left(f_{v}\right)$ for $v \in V$, and

(b) $\mathbf{C}(t) \models \Delta(f) \Rightarrow f \stackrel{\text { ae }}{=} f_{v}$ for some $v \in V$. 
Proof. The first part of this claim follows from the definitions of $f_{v}$ and $\Delta(x)$. For the second part choose a term $p\left(x_{1}, \ldots, x_{n}\right)$ and vertices $v_{1}, \ldots, v_{n} \in V$ such that

$$
f \stackrel{\text { ae }}{=} p^{\mathbf{B}}\left(f_{v_{1}}, \ldots, f_{v_{n}}\right) .
$$

Choose $i \in I$ such that $f(i)=p^{\mathbf{S}}\left(f_{v_{1}}(i), \ldots, f_{v_{n}}(i)\right)$. Then $f(i) \in S \cap \mu^{\mathbf{A}}$, which implies, by condition (iv) of the hypotheses of this lemma, that $f(i) \equiv$ $m$ for some $m \in M$. Finally, by condition (iii) it follows that $m=f_{v_{l}}(i)$ for some $l \leq n$ and so by Claim 2.2 we have that $f \stackrel{\text { ae }}{=} f_{v_{l}}$ as required.

Let $\sigma(x, y)$ be $(\tau(x) \rightarrow \tau(y))^{*}$, and let $\rho(x, y)$ be $\Delta(x) \wedge \Delta(y) \wedge \sigma(x, y)$.

Claim 2.5 $\{\llbracket g \not \equiv h \rrbracket: \mathbf{C}(t) \models \rho(g, h)\}$ is the set of all finite subsets of $I$.

Proof. First suppose $\mathbf{C}(t) \models \rho(g, h)$. Then $\llbracket \tau(g) \rightarrow \tau(h) \rrbracket=I$ and there are vertices $u, v \in V$ such that $g \stackrel{\text { ae }}{\equiv} f_{u}$ and $h \stackrel{\text { ae }}{=} f_{v}$ (by Claim 2.4). By the construction of $f_{u}$ and $f_{v}$ (in particular, $f_{w}^{-1}(e)$ is infinite for all $w \in V$ and $e \in E(\mathbf{G}))$ and the fact that every vertex is graph related to at least two vertices we must have that $u=v$. Thus $g \stackrel{\text { ae }}{=} h$ and so $\llbracket g \not \equiv h \rrbracket$ is finite.

Next let $J$ be a finite subset of $I$. Choose $v \in V, a \in M \cap \tau^{\mathbf{A}}$, and $b \in M \backslash \tau^{\mathbf{A}}$. Let $g, h \in M^{I}$ be defined by:

$$
\left.g\right|_{I-J}=\left.h\right|_{I-J}=\left.f_{v}\right|_{I-J}
$$

$g$ takes the value $b$ on $J$, and $h$ takes the value $a$ on $J$. Then $\mathbf{C}(t) \models \rho(g, h)$ and $\llbracket g \not \equiv h \rrbracket=J$.

Now we have enough expressive power to carry out the semantic embedding of $\mathbf{G}$ into $\mathbf{C}(t)$.

Claim 2.6 The two relations $\stackrel{\text { ae }}{=}$ and $\stackrel{\text { ae }}{=}$ are first order definable (with parameters) in the algebra $\mathbf{C}(t)$.

Proof. We leave it to the reader to check that $\mathbf{C}(t) \models f \stackrel{\text { ae }}{\equiv} g$ if and only if

$$
\mathbf{C}(t) \models \exists w, z\left[\rho(w, z) \wedge(f \not \equiv g \rightarrow w \not \equiv z)^{*}\right]
$$

and that $\mathbf{C}(t) \models f \stackrel{\text { ae }}{=} g$ if and only if

$$
\exists w, z\left[w \stackrel{\text { ae }}{=} z \wedge(w \equiv z \leftrightarrow f=g)^{*}\right] .
$$


Let $\operatorname{Disj}\left(x_{1}, y_{1}, x_{2}, y_{2}\right)$ be the formula $s\left(x_{1}, y_{1}, x_{2}, y_{2}\right) \approx x_{2}$. Then

$$
\mathbf{C}(t) \models \operatorname{Disj}\left(g_{1}, h_{1}, g_{2}, h_{2}\right) \Leftrightarrow \llbracket g_{1} \neq h_{1} \rrbracket \cap \llbracket g_{2} \neq h_{2} \rrbracket=\emptyset .
$$

Define $\operatorname{Edg}(x, y)$ to be

$$
\begin{gathered}
\forall w, z\left[w \stackrel{\text { ae }}{=} z \rightarrow \exists w_{1}, z_{1}\left(w_{1} \not z_{1} \wedge \operatorname{Disj}\left(w_{1}, z_{1}, w, z\right)\right.\right. \\
\left.\left.\wedge\left[w_{1} \not \approx z_{1} \rightarrow x \not \equiv y \wedge \tau(x) \wedge \tau(y)\right]^{*}\right)\right] .
\end{gathered}
$$

Then $(\Delta, \mathbf{E d g}, \stackrel{\text { ae }}{\equiv})$ semantically embeds $\mathbf{G}$ into $\mathbf{C}(t)$ since $\mathbf{G}$ is isomorphic to the structure $\left\langle\Delta^{\mathbf{C}(t)}, \operatorname{Edg}^{\mathbf{C}(t)}\right\rangle / \stackrel{\text { ae }}{=}$.

Let us quickly point out that a central result of Rubin [15] follows from the above.

COROLLARY 2.7 The theory of monadic algebras is undecidable.

Proof. Let $\mathbf{A}$ be the Boolean algebra of all subsets of $\omega$, let $M$ be the set $\{\{0,1,2\},\{3,4,5\},\{6,7\}, \ldots,\{2 n+6,2 n+7\}, \ldots\}$, Equiv be the equality relation, let $\mu(x)$ be the formula expressing " $x$ has exactly 2 or 3 atoms below it", and let $\tau(x)$ be the formula expressing " $x$ has exactly 3 atoms below it".

This algebra is easily seen to satisfy the hypothesis of Lemma 2.1, so with $K$ the class of Boolean algebras we see that $\mathrm{V}\left(K^{t}\right)$ is undecidable. The variety of monadic algebras is definitionally equivalent to this variety.

As another example of an application of the above lemma we have the following corollary. A unary term $t(x)$ of a variety $\mathcal{V}$ will be called constant (in $\mathcal{V}$ ) if $\mathcal{V} \models t(x) \approx t(y)$. $t$ will be called left invertible if for some term $l(x)$, $\mathcal{V} \models l(t(x)) \approx x$.

COROLLARY 2.8 Let $\mathcal{V}$ be a locally finite variety of multi-unary algebras. If $\mathrm{V}\left(\mathcal{V}^{t}\right)$ is not hereditarily undecidable then every nonconstant term of $\mathcal{V}$ must be left invertible, i.e., $\mathcal{V}$ is essentially a variety of $\mathbf{G}$-sets for some group $\mathbf{G}$. 
Proof. If $\mathrm{V}\left(\mathcal{V}^{t}\right)$ is not hereditarily undecidable then we must have that $\mathcal{V}$ is also not hereditarily undecidable. It was shown in [17] that if $\mathcal{V}$ is not hereditarily undecidable then for every two terms $f(x)$ and $g(x)$ of $\mathcal{V}$, we must have either $f \leq g$ or $g \leq f$, where $s \leq t$ if and only if there is some other term $h$ such that $\mathcal{V}=s(x) \approx h(t(x))$.

Now, if there is some nonconstant term $f(x)$ of $\mathcal{V}$ that is not left invertible, then choose such a term that is minimal with respect to the ordering $\leq$. Let $\mathbf{F}$ be the free algebra in $\mathcal{V}$ generated by the set $\left\{x_{1}, x_{2}, \ldots, x_{n}, \ldots\right\}$ and let A be the subalgebra of $\mathbf{F}$ generated by $\left\{x_{1}, x_{2}, f\left(x_{3}\right), \ldots, f\left(x_{n}\right), \ldots\right\}$. Let $M=\left\{f\left(x_{i}\right): i \geq 1\right\}$.

Define $a \leq b$ in $\mathbf{F}$ to mean that $a=g^{\mathbf{F}}(b)$ for some term $g$. Again, since $\mathcal{V}$ is locally finite the relation $\leq$ is first order definable in any algebra in $\mathcal{V}$. Define $\operatorname{Equiv}(x, y)$ to be $(x \leq y) \wedge(y \leq x)$. Then Equiv defines an equivalence relation on any algebra in $\mathcal{V}$ and in the case of $\mathbf{A}$, no two elements of $M$ are Equiv-related.

Let $C$ be the set of elements of $\mathbf{A}$ that are in the range of some constant valued term operation. Since $\mathcal{V}$ is locally finite, then $C$ is finite and is first order definable. We define $\mu(x)$ to be the formula that asserts that $x$ is not in $C$ and is minimal with respect to the ordering $\leq$ in the set $A \backslash C$. By our choice of the term $f$ it is clear that $\mathbf{A} \models \mu(a)$ if and only if $\operatorname{Equiv}\left(a, f\left(x_{n}\right)\right)$ is true in $\mathbf{A}$ for some $n$. We let $\tau(x)$ be the formula that expresses that there is some element $y$ such that $x \leq y$ and $y \not \leq x$. Then amongst the elements of $M$, only $f\left(x_{1}\right)$ and $f\left(x_{2}\right)$ satisfy $\tau$.

It is now easy to see, by the above lemma, that the variety $\mathrm{V}\left(\mathbf{A}^{t}\right)$ is hereditarily undecidable, and hence, so is the variety $\mathrm{V}\left(\mathcal{V}^{t}\right)$.

We will prove the converse to this in the next section.

\section{$3 \quad$ Decidability}

The following theorem is a generalization of McKenzie's unpublished result $[12]$ that the pure discriminator variety $\left[=\mathrm{V}\left(\omega^{t}\right)\right]$ is decidable.

Definition 3.1 A finite structure is said to be homogeneous if every isomorphism between substructures extends to an automorphism of the structure. For $\mathcal{K}$ a class of structures, we say that $\mathcal{K}$ is homogeneous if every finite member of $\mathcal{K}$ is. 
THEOREM 3.2 Let $\mathcal{K}$ be a homogeneous, locally finite, finitely axiomatizable universal class with a finite language. Then the class $\Gamma^{a}(\mathcal{K})$ is decidable.

Proof. Using the Compactness Theorem, it follows that since $\mathcal{K}$ is locally finite, universal and of finite type, then for each $n$ there is a finite upper bound to the size of all $n$-generated members of $\mathcal{K}$. As we shall see in the next paragraph, there is a recursive function $\sigma(n)$ that provides this bound.

Given $n$ and $m$, let $T_{m, n}$ be the set of all $n$-ary terms of $\mathcal{K}$ of length at most $m$. Since $\mathcal{K}$ is of finite type, it follows that $T_{m, n}$ is finite. Furthermore, since $\mathcal{K}$ is locally finite, there must be some $m$ such that $\mathcal{K}$ proves that for every $n$-tuple of elements $\bar{x}$, the set of elements obtained by applying the terms from $T_{m+1, n}$ to $\bar{x}$ is equal to the set of elements obtained by applying the terms from $T_{m, n}$ to $\bar{x}$. Since $\mathcal{K}$ is finitely axiomatizable we can effectively find this $m$, by enumerating all proofs in $\mathcal{K}$ and stopping when a proof of a statement as above is seen. Then $\sigma(n)$ can be set to be the size of $T_{m, n}$.

From the recursiveness of $\sigma$ we can easily show that the universal theory of $\mathcal{K}$ is decidable. Given a universal sentence $\phi$ of $\mathcal{K}$, there is a quantifier free formula $q\left(x_{1}, \ldots, x_{n}\right)$, for some $n$, such that $\phi=\forall \bar{x} q(\bar{x})$. Since $\mathcal{K}$ is closed under subalgebras, it follows that $\phi$ is true in all algebras in $\mathcal{K}$ if and only if it is true in all $n$-generated algebras in $\mathcal{K}$. Since $\mathcal{K}$ is of finite type and finitely axiomatizable, and since we can effectively bound the size of all $n$-generated algebras in $\mathcal{K}$ (using $\sigma$ ) we can effectively construct, up to isomorphism, all $n$-generated algebras in $\mathcal{K}$ and check whether or not $\phi$ holds in all of them.

For a given $n$, we can also effectively compute quantifier free formulas $\delta_{n, i}\left(x_{1}, \ldots, x_{n}\right), 1 \leq i \leq \lambda(n)$, which describe the various possible quantifier free types of $n$-tuples of elements from algebras in $\mathcal{K}$. Here $\lambda(n)$ is the function whose value is the number of these quantifier free $n$-types. From the previous paragraph we see that $\lambda$ is a recursive function.

Corresponding to each of the formulas $\delta_{n, i}$ is an algebra $\mathbf{S}_{n, i}$ generated by a sequence of elements $1, \ldots, n$ which satisfy $\delta_{n, i}$. We will write $\mathbf{S}_{n-1, i} \hookrightarrow \mathbf{S}_{n, j}$ to mean there is an embedding from $\mathbf{S}_{n-1, i}$ to $\mathbf{S}_{n, j}$ which is the identity on the set $\{1, \ldots, n-1\}$. For $p\left(x_{1}, \ldots, x_{n}\right)$ a term let $p(\vec{n})$ denote $p(1, \ldots, n)$.

Now for each first-order L-formula $\phi\left(x_{1}, \ldots, x_{n}\right)$ we define inductively a formula $\Phi\left(X_{1}, \ldots, X_{\lambda(n)}\right)$ in the language of Boolean algebras with distinguished ideals $J_{n, i}$ as follows:

(1) if $\phi$ is atomic, say $\phi$ is

$$
p\left(x_{1}, \ldots, x_{n}\right) \approx q\left(x_{1}, \ldots, x_{n}\right),
$$


then let $\Phi\left(X_{1}, \ldots, X_{\lambda(n)}\right)$ be

$$
\bigvee\left\{X_{i}: \mathbf{S}_{n, i} \models p(\vec{n})=q(\vec{n})\right\}=1 ;
$$

(2) if $\phi$ is $\neg \theta$ and $\Theta$ is given then let $\Phi$ be $\neg \Theta$;

(3) if $\phi$ is $\theta_{1} \wedge \theta_{2}$ and $\Theta_{1}, \Theta_{2}$ are given then let $\Phi$ be $\Theta_{1} \wedge \Theta_{2}$;

(4) if $\phi\left(x_{1}, \ldots, x_{n-1}\right)$ is $\exists x_{n} \theta\left(x_{1}, \ldots, x_{n}\right)$ and $\Theta\left(X_{1}, \ldots, X_{\lambda(n)}\right)$ is given then let $\Phi\left(X_{1}, \ldots, X_{\lambda(n-1)}\right)$ be:

$$
\begin{gathered}
\exists Y_{1}, \ldots, Y_{\lambda(n)}\left[\operatorname{Part}\left(Y_{1}, \ldots, Y_{\lambda(n)}\right) \wedge \bigwedge_{1 \leq i \leq \lambda(n)} Y_{i} \in J_{n, i} \wedge\right. \\
\left.\bigwedge_{1 \leq j \leq \lambda(n-1)}\left(X_{j}=\bigvee_{\mathbf{s}_{n-1, j} \hookrightarrow \mathbf{s}_{n, i}} Y_{i}\right) \wedge \Theta\left(Y_{1}, \ldots, Y_{\lambda(n)}\right)\right],
\end{gathered}
$$

where the formula Part above says that $Y_{1}, \ldots, Y_{\lambda(n)}$ partition unity.

Claim 3.3 Let $\mathbf{A} \in \Gamma^{a}(\mathcal{K})$, say $\mathbf{A}$ is a Boolean product over the Boolean space $X(\mathbf{A})$ of the algebras $\mathbf{A}_{x}$ from $\mathcal{K}$, for $x \in X(\mathbf{A})$. Let $\mathbf{B}$ be the Boolean algebra of clopen subsets of $X(\mathbf{A})$ along with the distinguished ideals $J_{n, i}$, for $n \geq 1$ and $i \leq \lambda(n)$, where $J_{n, i}$ is the ideal of $\mathbf{B}$ that corresponds (using Stone duality) to the open set

$$
\left\{x \in X(\mathbf{A}): \text { there is an embedding of } \mathbf{S}_{n, i} \text { into } \mathbf{A}_{x}\right\}
$$

of $X(\mathbf{A})$. Then for any first-order L-formula $\phi(\bar{x})$ and parameters $\bar{a}$ from A the following holds:

$$
\mathbf{A} \models \phi\left(a_{1}, \ldots, a_{n}\right) \Leftrightarrow \mathbf{B}=\Phi\left(\llbracket \delta_{n, 1}(\bar{a}) \rrbracket, \ldots, \llbracket \delta_{n, \lambda(n)}(\bar{a}) \rrbracket\right) .
$$

\section{ProOF.}

This variant of the well-known Feferman-Vaught theorem is proved by induction on the complexity of the formula $\phi$. We will only present the proof of the following case in the induction: $\phi\left(x_{1}, \ldots, x_{n-1}\right)$ is equal to $\exists y \theta\left(x_{1}, \ldots, x_{n}, y\right)$ for some formula $\theta$ for which (1) applies. 
Suppose that $\mathbf{A} \models \phi\left(a_{1}, \ldots, a_{n-1}\right)$ for some elements $a_{i}$ from $A$. Then there is an element $b$ from $A$ with $\mathbf{A} \models \theta\left(a_{1}, \ldots, a_{n-1}, b\right)$. By the inductive hypothesis we have

$$
\mathbf{B} \models \Theta\left(\llbracket \delta_{n, 1}(\bar{a}, b) \rrbracket, \ldots, \llbracket \delta_{n, \lambda(n)}(\bar{a}, b) \rrbracket\right) .
$$

Since the $\llbracket \delta_{n, i}(\bar{a}, b) \rrbracket$ 's partition $B$ and for each $i, \llbracket \delta_{n, i}(\bar{a}, b) \rrbracket \in J_{n, i}$, we need only prove that for each $j \leq \lambda(n-1)$, $\llbracket \delta_{n-1, j}(\bar{a}) \rrbracket$ is equal to the union of the $\llbracket \delta_{n, i}(\bar{a}, b) \rrbracket$ such that $\mathbf{S}_{n-1, j} \hookrightarrow \mathbf{S}_{n, i}$ in order to establish that $\mathbf{B} \models$ $\Phi\left(\llbracket \delta_{n-1,1}(\bar{a}) \rrbracket, \ldots, \llbracket \delta_{n-1, \lambda(n-1)}(\bar{a}) \rrbracket\right)$.

If $x \in \llbracket \delta_{n-1, j}(\bar{a}) \rrbracket$ then for some $i \leq \lambda(n)$ we have

$$
\mathbf{A}_{x} \models \delta_{n, i}\left(\left(a_{1}\right)_{x}, \ldots,\left(a_{n-1}\right)_{x}, b_{x}\right) .
$$

So it follows that $\mathbf{S}_{n-1, j} \hookrightarrow \mathbf{S}_{n, i}$ since

$$
\mathbf{A}_{x} \models \delta_{n-1, j}\left(\left(a_{1}\right)_{x}, \ldots,\left(a_{n-1}\right)_{x}\right) .
$$

Conversely, suppose that $x$ is in $\llbracket \delta_{n, i}(\bar{a}, b) \rrbracket$ for some $i$ such that $\mathbf{S}_{n-1, j} \hookrightarrow$ $\mathbf{S}_{n, i}$. Then it is immediate that $\mathbf{A}_{x} \models \llbracket \delta_{n-1, j}\left(\left(a_{1}\right)_{x}, \ldots,\left(a_{n-1}\right)_{x}\right) \rrbracket$ and so $x \in \llbracket \delta_{n-1, j}(\bar{a}) \rrbracket$.

Now, if

$$
\mathbf{B} \models \Phi\left(\llbracket \delta_{n-1,1}(\bar{a}) \rrbracket, \ldots, \llbracket \delta_{n-1, \lambda(n-1)}(\bar{a}) \rrbracket\right)
$$

then there are clopen sets of $\mathbf{B}, Y_{1}, \ldots, Y_{\lambda(n)}$ which witness this fact. For $x \in Y_{i}$, there is a unique $j \leq \lambda(n-1)$ such that $x \in \llbracket \delta_{n-1, j}(\bar{a}) \rrbracket$. By the third clause of the formula $\Phi$, it follows that $\mathbf{S}_{n-1, j} \hookrightarrow \mathbf{S}_{n, i}$. By the homogeneity of $\mathcal{K}$ and the fact that $Y_{i} \in J_{n, i}$ it follows that there is some element $b$ of $A$ such that $x \in \llbracket \delta_{n, i}(\bar{a}, b) \rrbracket$.

Thus the collection of sets $\llbracket \delta_{n, i}(\bar{a}, b) \rrbracket$, for $b \in A$, forms a clopen cover of $Y_{i}$ and so there are elements $b_{1}, \ldots, b_{m}$ such that

$$
Y_{i} \subseteq \bigcup_{j \leq m} \llbracket \delta_{n, i}\left(\bar{a}, b_{j}\right) \rrbracket .
$$

Using the "patchwork" property of Boolean products, we can find an element $c_{i}$ of $\mathbf{A}$ such that

$$
Y_{i} \subseteq \bigcup_{j \leq m}\left(\llbracket c_{i}=b_{j} \rrbracket \cap \llbracket \delta_{n, i}\left(\bar{a}, b_{j}\right) \rrbracket\right) .
$$


From this we can conclude that $Y_{i} \subseteq \llbracket \delta_{n, i}\left(\bar{a}, c_{i}\right) \rrbracket$.

We can patch together the $c_{i}$ to get an element $c$ in $\mathbf{A}$ such that $Y_{i} \subseteq \llbracket c=$ $c_{i} \rrbracket$, and so $Y_{i} \subseteq \llbracket \delta_{n, i}(\bar{a}, c) \rrbracket$ for each $i \leq \lambda(n)$. Since the $Y_{i}$ and the $\llbracket \delta_{n, i}(\bar{a}, c) \rrbracket$ both partition $X(\mathbf{A})$ we must have that $Y_{i}=\llbracket \delta_{n, i}(\bar{a}, c) \rrbracket$ for all $i$. Therefore

$$
\mathbf{B} \models \Theta\left(\llbracket \delta_{n, 1}(\bar{a}, c) \rrbracket, \ldots, \llbracket \delta_{n, \lambda(n)}(\bar{a}, c) \rrbracket\right)
$$

and so by induction,

$$
\mathbf{A} \models \theta(\bar{a}, c)
$$

and hence $\mathbf{A} \models \phi(\bar{a})$.

An immediate consequence of the above claim is that if a sentence $\phi$ fails in some member of $\Gamma^{a}(\mathcal{K})$ then it fails in some member of $\Gamma^{a}(\mathcal{K})$ in which all the stalks have size bounded by some finite number $N$ (since $\Phi$ involves only finitely many of the symbols $J_{n, i}$ ). This number $N$ can be effectively calculated since, as noted above, the universal theory of $\mathcal{K}$ is decidable. Thus $\phi$ holds in $\Gamma^{a}(\mathcal{K})$ iff it holds in $\Gamma^{a}\left(\mathcal{K}_{\leq N}\right)$, where $\mathcal{K}_{\leq N}$ is the class of algebras in $\mathcal{K}$ of size $\leq N$. Given $\mathcal{K}_{\leq N}$ we know from [4, Theorem 6.1] how to decide the theory of $\Gamma^{a}\left(\mathcal{K}_{\leq N}\right)$. Consequently the theory of $\Gamma^{a}(\mathcal{K})$ is decidable.

COROLLARY 3.4 Let $\mathcal{K}$ be a homogeneous, locally finite, finitely axiomatizable universal class with a finite language. Then $\mathrm{V}\left(\mathcal{K}^{t}\right)$ is decidable.

Proof. Observe that $\mathcal{K}^{t}$ satisfies the hypotheses of Theorem 3.2 since $\mathcal{K}$ does. Since $\mathrm{V}\left(\mathcal{K}^{t}\right)=\mathrm{I} \Gamma^{a}\left(\mathcal{K}^{t}\right)$, Theorem 3.2 applies.

COROLLARY 3.5 Let $\mathcal{V}$ be a locally finite variety of unary algebras in a finite language. Then the following are equivalent:

(i) $\mathrm{V}\left(\mathcal{V}^{t}\right)$ is decidable;

(ii) $\mathrm{V}\left(\mathcal{V}^{t}\right)$ is not hereditarily undecidable;

(iii) Every nonconstant unary term of $\mathcal{V}$ is left invertible.

Proof. Certainly if $\mathrm{V}\left(\mathcal{V}^{t}\right)$ is decidable then it is not hereditarily undecidable. We know from Corollary 2.8 that if $\mathrm{V}\left(\mathcal{V}^{t}\right)$ is not hereditarily undecidable then every nonconstant unary term of $\mathcal{V}$ is left invertible. 
Finally, suppose that every nonconstant unary term of $\mathcal{V}$ is left invertible. Then $\mathcal{V}$ is essentially equal to a variety of $\mathbf{G}$-sets with some distinguished constants for some finite group $\mathbf{G}$ and so $\mathcal{V}$ is homogeneous and finitely axiomatizable. From the above corollary the decidability of $\mathrm{V}\left(\mathcal{V}^{t}\right)$ follows.

COROLLARY 3.6 Let $\mathcal{V}$ be a variety of mono-unary algebras in the language $\mathbf{L}=\{f\}$. Then the following are equivalent:

(i) $\mathrm{V}\left(\mathcal{V}^{t}\right)$ is decidable;

(ii) $\mathrm{V}\left(\mathcal{V}^{t}\right)$ is not hereditarily undecidable;

(iii) $\mathcal{V}$ is axiomatized by one of the following equations: $f^{n}(x) \approx x$, for some $n ; f(x) \approx f(y) ; x \approx y$.

Proof. If the variety $\mathcal{V}$ is locally finite, then the previous corollary applies. If $\mathcal{V}$ is not locally finite, then it must be the class of all monounary algebras. This variety contains many locally finite subvarieties $\mathcal{W}$ with $\mathrm{V}\left(\mathcal{W}^{t}\right)$ hereditarily undecidable (just use Corollary 2.8), and hence, $\mathrm{V}\left(\mathcal{V}^{t}\right)$ is also hereditarily undecidable.

\section{Strongly Abelian Varieties}

The class of strongly Abelian algebras was first defined by Ralph McKenzie in [13]. The significance of these algebras, especially in the role they play in the classification of finite algebras and locally finite varieties has been demonstrated in $[10,13,14]$. In this section we extend corollary 3.5 to a characterization of the locally finite strongly Abelian varieties $\mathcal{V}$ such that $\mathrm{V}\left(\mathcal{V}^{t}\right)$ is decidable.

Definition 4.1 An algebra is called strongly Abelian if for all terms $t(x, \bar{y})$, for all $a, b, \bar{c}, \bar{d}$ and $\bar{e}$,

$$
t(a, \bar{c})=t(b, \bar{d}) \longrightarrow t(a, \bar{e})=t(b, \bar{e}) .
$$

A variety is called strongly Abelian if each of its members is. 
Unary algebras are easily seen to be strongly Abelian. Rectangular bands (i.e., semigroups satisfying $x y x \approx x$ ) are examples of non-unary strongly Abelian algebras.

In order to characterize the locally finite strongly Abelian varieties $\mathcal{V}$ with $\mathrm{V}\left(\mathcal{V}^{t}\right)$ decidable we must first give a description of the locally finite strongly Abelian varieties of finite type that are decidable. This was first done in [19], but the reader may wish to consult [14] or [9] for details of this characterization. To understand this characterization we must introduce a method for constructing strongly Abelian algebras from multi-sorted unary algebras.

Let $\mathrm{L}$ be a language for $k$-sorted unary algebras. We construct a language $\mathrm{L}_{k}$ of (one-sorted) algebras as follows: for each sequence $f_{1}, \ldots, f_{k}$ of function symbols or unary projections of $\mathrm{L}$, where the sort of $f_{i}$ is $i$, we include in $\mathrm{L}_{k}$ the $k$-ary function symbol $\left[f_{1}, \ldots, f_{k}\right]$. Note that if $\mathbf{L}$ is finite, then so is $\mathbf{L}_{k}$.

For $\mathbf{A}$ an $\mathrm{L}$ algebra with universes $A_{1}, \ldots, A_{k}$, we define an $\mathrm{L}_{k}$ algebra $\mathbf{A}[k]$ in the following way: the universe of $\mathbf{A}[k]$ is $A_{1} \times \cdots \times A_{k}$ and for each $\left[f_{1}, \ldots, f_{k}\right]$ from $\mathrm{L}_{k}$, define

$$
\left[f_{1}, \ldots, f_{k}\right]^{\mathbf{A}[k]}\left(\vec{a}_{1}, \ldots, \vec{a}_{k}\right)=\left\langle f_{1}^{\mathbf{A}}\left(a_{1}^{i_{1}}\right), \ldots, f_{k}^{\mathbf{A}}\left(a_{k}^{i_{k}}\right)\right\rangle,
$$

where $\overrightarrow{a_{i}}=\left\langle a_{i}^{1}, \ldots, a_{i}^{k}\right\rangle$ for all $i \leq k$ and for all $j \leq k$, the sort of the domain of $f_{j}^{\mathbf{A}}$ is $i_{j}$.

The algebras $\mathbf{A}$ and $\mathbf{A}[k]$ are closely related, in particular any subalgebra or automorphism of one of these algebras is determined in a natural way by a subalgebra or automorphism from the other.

If $\mathcal{V}$ is a variety of $k$-sorted algebras of type $\mathrm{L}$ then it can be shown that the class $\mathcal{V}[k]=\{\mathbf{B}: \mathbf{B}$ is isomorphic to $\mathbf{A}[k]$ for some $\mathbf{A}$ from $\mathcal{V}\}$ is a variety of $\mathrm{L}_{k}$ algebras.

Definition 4.2 A variety of $k$-sorted unary algebras is said to be linear if for all nonconstant unary terms $t(x)$ and $s(x)$, there is a term $h(y)$ of the appropriate sort such that $\mathcal{W}=t(x) \approx h(s(x))$ or $\mathcal{W}=s(x) \approx h(t(x))$.

The following theorem gives a complete characterization of the decidable locally finite strongly Abelian varieties of finite type. The proof of this theorem can be found in [14]. 
THEOREM 4.3 Let $\mathcal{V}$ be a locally finite strongly Abelian variety of finite type. Then $\mathcal{V}$ is decidable if and only if $\mathcal{V}$ is term equivalent to $\mathcal{W}[k]$ for some $k$ and some $k$-sorted, unary, linear variety $\mathcal{W}$.

We are now ready to state our characterization of the decidable varieties of the form $\mathrm{V}\left(\mathcal{V}^{t}\right)$, where $\mathcal{V}$ is a locally finite strongly Abelian variety of finite type.

THEOREM 4.4 Let $\mathcal{V}$ be a locally finite strongly Abelian variety of finite type. Then $\mathrm{V}\left(\mathcal{V}^{t}\right)$ is decidable if and only if $\mathcal{V}$ is term equivalent to a variety $\mathcal{W}[k]$, where $\mathcal{W}$ is a $k$-sorted unary variety such that every term of $\mathcal{W}$ is either constant or left invertible.

Proof. One direction of this theorem is straightforward and follows from Corollary 3.2, for if every term $s(x)$ of $\mathcal{W}$ is either constant or left invertible, then every finite algebra in $\mathcal{W}$ is seen to be homogeneous. This carries over to the finite algebras in the variety $\mathcal{W}[k]$ and hence to $\mathcal{V}$.

For the converse, if $\mathrm{V}\left(\mathcal{V}^{t}\right)$ is decidable, then by Theorem 4.3, we may assume that $\mathcal{V}$ is equal to $\mathcal{W}[k]$ for some $k$-sorted, unary, linear variety $\mathcal{W}$. We will use Lemma 2.1 to establish undecidability under the assumption that there is some nonconstant term $s(x)$ of $\mathcal{W}$ that is not left invertible. This part of the proof is modeled after the proof of Corollary 2.8.

For any two terms $f(x)$ and $g(y)$ of $\mathcal{W}$, we define $f \leq g$ to mean that there is some term $h(z)$ of $\mathcal{W}$, of the appropriate sort, such that

$$
\mathcal{W} \models f(x) \approx h(g(x)) \text {. }
$$

Note that $f \leq g$ implies that the variables $x$ and $y$ are of the same sort. The relation $\leq$ is clearly transitive and reflexive and so the relation $x \cong y$ defined by $x \leq y$ and $y \leq x$ is an equivalence relation on the set of unary terms of $\mathcal{W}$.

The linearity assumption on $\mathcal{W}$ implies that for all nonconstant terms $f(x)$ and $g(x)$, either $f \leq g$ or $g \leq f$. Furthermore, since $\mathcal{W}$ is locally finite, it follows that there are terms minimal with respect to $\leq$ amongst the nonconstant unary terms. Clearly, a term $f$ is maximal with respect to $\leq$ if and only if it is left invertible. Choose a nonconstant term $f(x)$ that is minimal with respect to $\leq$ and not left invertible. We may assume without loss of generality that the sort of the variable $x$ is 1 and that the sort of $f$ 
is either 1 or 2 . There are two cases to consider, we will examine the case where the sort of $f$ is 2 . The remaining case can be handled in a similar way.

We wish to use Lemma 2.1 to establish the undecidability of $\mathrm{V}\left(\mathcal{V}^{t}\right)$ and so we must find a suitable algebra $\mathbf{A}$ to work with. To do this, we will describe an algebra $\mathbf{B}$ in $\mathcal{W}$ and let $\mathbf{A}$ be $\mathbf{B}[k]$, expanded by naming a single element from the universe. For each $2 \leq i \leq k$, let $X_{i}=\left\{x_{0}^{i}\right\}$ and let $X_{1}=\left\{x_{j}^{1}: j \geq 0\right\}$. Let $\mathbf{F}$ be the free algebra in $\mathcal{W}$ generated by the sequence $\left\langle X_{1}, X_{2}, \ldots, X_{k}\right\rangle$ and let $\mathbf{B}$ be the subalgebra of $\mathbf{F}$ generated by the set

$$
\left\{x_{0}^{1}, \ldots, x_{0}^{k}\right\} \cup\left\{f\left(x_{j}^{1}\right): j \geq 1\right\} \cup\left\{x_{1}^{1}, x_{2}^{1}\right\} .
$$

We set $\mathbf{A}$ to be the algebra $\mathbf{B}[k]$, expanded by naming the element $\left\langle x_{0}^{1}, x_{0}^{2}, \ldots, x_{0}^{k}\right\rangle$. For convenience, we will denote this element by $c$. For each $j>0$, let $m_{j}$ be the element $\left\langle x_{0}^{1}, f\left(x_{j}^{1}\right), x_{0}^{3}, \ldots, x_{0}^{k}\right\rangle$ in $\mathbf{B}[k]$ and let $M=\left\{m_{j}: j>0\right\}$.

In order to define the formulas Equiv, $\mu$ and $\tau$ needed in Lemma 2.1, we will first define some auxiliary formulas. For two elements $a$ and $b$ from $B$, we write $a \leq b$ if there is some term $h(x)$ of the appropriate sort such that $h^{\mathbf{B}}(b)=a$, and write $a \cong b$ if $a \leq b$ and $b \leq a$. Since $\mathcal{W}$ is locally finite then both of these relations are definable by first order formulae in $\mathbf{B}$.

For each $i \leq k$, there is a formula $x={ }_{i} y$ in the language of $\mathcal{W}[k]$ such that

$$
\mathbf{A} \models u={ }_{i} v \quad \text { if and only if } \quad u_{i}=v_{i} .
$$

Namely, set $x={ }_{i} y$ to be the formula

$$
\left[x_{1}, x_{2}, \ldots, x_{k}\right](x, x, \ldots, \stackrel{i}{y}, x, \ldots, x)=x,
$$

where $x_{j}$ is a variable of $\mathcal{W}$ of sort $j$.

For each $i, j \leq k$, let $x \leq_{i j} y$ be a first order formula in the language of $\mathcal{W}[k]$ such that

$$
\mathbf{A} \models u \leq_{i j} v \quad \text { if and only if } \quad \mathbf{B} \models u_{i} \leq v_{j} .
$$

Since $\mathcal{W}$ is locally finite, then this condition is equivalent in $\mathcal{W}[k]$ to a finite disjunction of formulae of the form:

$$
\left[x_{1}, x_{2}, \ldots, x_{i-1}, h(z), x_{i+1}, \ldots, x_{k}\right](x, x, \ldots, \stackrel{j}{y}, x, \ldots, x)=x
$$


where $h$ is a term of sort $i, z$ is a variable of sort $j$ and for each $l, x_{l}$ is a variable of sort $l$.

Let $x \cong_{i j} y$ be the formula $\left(x \leq_{i j} y\right) \wedge\left(y \leq_{j i} x\right)$. Then from the previous paragraph, it follows that $\mathbf{A} \models u \cong_{i j} v$ if and only if $u_{i} \cong v_{j}$ in $\mathbf{B}$.

For $i \leq k$, let $\mathcal{C}_{i}$ be the set of all constant terms of $\mathcal{W}$ of sort $i$. Since $\mathcal{W}$ is locally finite it follows that the property "the $i$ th component of an element $x$ of $\mathbf{A}$ is in the range of some constant term operation of $\mathbf{B}$ " is expressible as a first order formula $\mathcal{C}_{i}(x)$. In fact this property is equivalent to a finite disjunction of formulae of the form:

$$
\left[x_{1}, x_{2}, \ldots, x_{i-1}, s(z), x_{i+1}, \ldots, x_{k}\right](x, x, \ldots, x)=x,
$$

where $s$ belongs to $\mathcal{C}_{i}$ and for each $j, x_{j}$ is a variable of sort $j$.

Now we are in a position to define the formulae that are needed in Lemma 2.1. Let $\operatorname{Equiv}(x, y)$ be the formula

$$
\bigwedge_{i \neq 2}\left(x={ }_{i} y\right) \wedge\left(x \cong_{22} y\right)
$$

Let $\mu(x)$ be the formula

$$
\bigwedge_{i \neq 2}\left(x={ }_{i} c\right) \wedge \bigwedge_{i \leq k}\left(x \mathbb{Z}_{2 i} c\right) \wedge \neg \mathcal{C}_{2}(x) \wedge \bigwedge_{i \leq k} \forall y\left(y \leq_{i 2} x \rightarrow\left[y \cong_{i 2} x \vee \mathcal{C}_{i}(y)\right]\right) .
$$

Let $\tau(x)$ be the formula

$$
\exists w\left[\left(x \leq_{21} w\right) \wedge\left(w \mathbb{L}_{12} x\right)\right] .
$$

Claim 4.5 The algebra $\mathbf{A}$, the set $M$ and the formulae Equiv, $\mu$ and $\tau$ satisfy the conditions of Lemma 2.1 .

PROOF.

(i) Let $\equiv$ be the binary relation Equiv $^{\mathbf{A}}$ restricted to the subalgebra $\mathbf{S}$ generated by $M$. Then for $m_{i}$ and $m_{j}$ from the set $M$, if $i \neq j$ then $m_{i} \approx_{22} m_{j}$, since $\mathcal{W}$ does not satisfy any equation of the form $f\left(x_{i}^{1}\right) \approx$ $h\left(f\left(x_{j}^{1}\right)\right)$ for any term $h$. Thus, if $u$ and $v$ are distinct elements from $M$ then $\mathbf{A} \models u \not \equiv v$. $\equiv$ clearly defines an equivalence relation since it is a conjunction of equivalence relations. It is also invariant under automorphisms of $\mathbf{S}$, since it is defined by a quantifier free formula. 
(ii) Let $\alpha$ be a bijection between two finite subsets $U$ and $V$ of $M$. This induces a bijection between the sets of free generators $\left\{x_{j}^{1}: m_{j}\right.$ in $\left.U\right\}$ and $\left\{x_{j}^{1}: m_{j}\right.$ in $\left.V\right\}$ and this bijection can be extended in a natural way to an automorphism $\beta$ of the free algebra $\mathbf{F}$ that fixes all other generators. Now the restriction of $\beta$ to the subalgebra $\mathbf{B}$ determines an automorphism, $\gamma$, of $\mathbf{A}$. It is not hard to see that the restriction of $\gamma$ to $\mathbf{S}$ is an automorphism that extends $\alpha$.

(iii) Let $J \subseteq M$ be finite and let $m_{j}$ be an element from $M$ that is $\equiv$ related to some member $v$ of the subalgebra of $\mathbf{A}$ generated by $J$. By the definition of $\equiv$, it follows that $m_{j}$ belongs to this subalgebra. From this it is easy to show that $m_{j}$ must actually belong to $J$.

(iv) Let $m$ belong to $M$ and $a \in S$ with $a \equiv m$. To show that $\mathbf{A} \models \mu(a)$ it will suffice to show that $\mathbf{A}=\mu(m)$, since $\mu$ is invariant under $\equiv$. This follows, by the definition of $M$, and by our choice of the term $f$.

Conversely, if $a \in S$ and $\mathbf{A} \models \mu(a)$, then $a=\left\langle x_{0}^{1}, h\left(x_{j}^{1}\right), x_{0}^{3}, \ldots, x_{0}^{k}\right\rangle$ for some nonconstant term $h$ and some $j$. Furthermore, $h$ must be minimal with respect to $\leq$ amongst the nonconstant terms. From our choice of $f$, it then follows that $h \cong f$ and so $h\left(x_{j}^{1}\right) \cong f\left(x_{j}^{1}\right)$. Thus, $a \equiv m_{j}$.

(v) It is not hard to see that the elements $m_{1}$ and $m_{2}$ both satisfy $\tau$, as witnessed by the elements $\left\langle x_{1}^{1}, x_{0}^{2}, \ldots, x_{0}^{k}\right\rangle$ and $\left\langle x_{2}^{1}, x_{0}^{2}, \ldots, x_{0}^{k}\right\rangle$ respectively. Also, no other element of $M$ satisfies $\tau$ since, for $j>2$, if $e$ is in $B$ and $f\left(x_{j}^{1}\right) \leq e$, then we must also have $e \leq f\left(x_{j}^{1}\right)$.

(vi) From the definitions of $\equiv, \mu$ and $\tau$, it follows that $\mu$ and $\tau$ are closed under $\equiv$.

We can now conclude that the discriminator variety $\mathrm{V}\left(\mathbf{A}^{t}\right)$ is hereditarily undecidable and hence so is the variety $\mathrm{V}\left(\mathcal{V}^{t}\right)$.

\section{Conclusion}

As noted above, if $\mathcal{K}$ is a class of similar algebras having an undecidable theory, then the discriminator variety $\mathrm{V}\left(\mathcal{K}^{t}\right)$ is also undecidable. So, using 
the characterization of decidable locally finite varieties found in [14] and the main result of [1], it follows that if $\mathcal{V}$ is a locally finite variety such that $\mathrm{V}\left(\mathcal{V}^{t}\right)$ is decidable, then $\mathcal{V}$ must be the varietal product of a strongly Abelian variety and an affine variety. In the previous section we have seen that the strongly Abelian factor must arise, in a natural way, from a variety of multi-sorted unary algebras such that every nonconstant term is left (and hence right) invertible.

Thus in order to completely characterize those locally finite varieties of finite type $\mathcal{V}$ with $\mathrm{V}\left(\mathcal{V}^{t}\right)$ decidable, we must do this for the locally finite affine varieties. As we have seen, if the finite algebras in such a variety are homogeneous, then $\mathrm{V}\left(\mathcal{V}^{t}\right)$ is decidable. It has been pointed out to us by Wilfred Hodges and Emil Kiss that the variety of all left $\mathbf{R}$-modules, for a finite ring $\mathbf{R}$ has this homogeneity property if and only if $\mathbf{R}$ is semi-simple. Hence, if $\mathbf{R}$ is finite and semi-simple, it follows from Lemma 3.2 that $\mathbf{V}\left(\mathcal{M}_{\mathbf{R}}^{t}\right)$ is decidable.

Recently, Valeriote and Willard [18] have proved that the converse is true, namely, for a finite ring $\mathbf{R}$, if the variety $\mathrm{V}\left(\mathcal{M}_{\mathbf{R}}^{t}\right)$ is decidable then $\mathbf{R}$ is semi-simple.

Their proof relies on a powerful new semantic embedding developed by Willard, similar to our Lemma 2.1. Using this tool, Willard [20] has been able to completely characterize those locally finite universal classes of unary algebras $\mathcal{U}$ of finite type such that $\mathrm{V}\left(\mathcal{U}^{t}\right)$ is decidable.

\section{References}

[1] S. Burris. Iterated discriminator varieties have undecidable theories. Algebra Universalis, 21:54-61, 1985.

[2] S. Burris and R. McKenzie. Decidability and Boolean Representations, volume 246 of Memoirs of the American Mathematical Society. American Mathematical Society, 1981.

[3] S. Burris and H.P. Sankappanavar. A Course in Universal Algebra. Springer-Verlag, 1981.

[4] S. Burris and H. Werner. Sheaf constructions and their elementary properties. Trans. Amer. Math. Soc., 248:269-309, 1979. 
[5] S. Comer. Elementary properties of structures of sections. Bol. Soc. Math. Mexicana, 19:78-85, 1974.

[6] S. Comer. Monadic algebras with finite degree. Algebra Universalis, 5:315-327, 1975.

[7] Yu. L. Ershov. Decidability of the elementary theory of relatively complemented distributive lattices and the theory of filters. Algebra $i$ Logika, 3:17-38, 1964.

[8] Yu. L. Ershov. On the elementary theory of Post varieties. Algebra $i$ Logika, 6:7-15, 1967.

[9] B. Hart and M. Valeriote. A structure theorem for strongly abelian varieties with few models. The Journal of Symbolic Logic, 56:832-852, 1991.

[10] D. Hobby and R. McKenzie. The Structure of Finite Algebras, volume 76 of Contemporary Mathematics. American Mathematical Society, 1988.

[11] R. McKenzie. On the spectra, and negative solution of the decision problem for identities having a finite nontrivial model. Journal of Symbolic Logic, 40:186-196, 1975.

[12] R. McKenzie. Decidability of the theory of the pure discriminator variety. University of California, Berkeley (notes by S. Burris), 1976.

[13] R. McKenzie. Finite forbidden lattices. In Universal Algebra and Lattice Theory, volume 1004 of Springer Lecture Notes. Springer-Verlag, 1983.

[14] R. McKenzie and M. Valeriote. The Structure of Locally Finite Decidable Varieties, volume 79 of Progress in Mathematics. Birkhäuser Boston, 1989.

[15] M. Rubin. The theory of Boolean algebras with a distinguished subalgebra is undecidable. Ann. Sci. Univ. Clermnont. No. 60 Math No. 13.

[16] A. Tarski. Arithmetical classes and types of Boolean algebras. Bull. Amer. Math. Soc., 55:64, 1949. 
[17] M. Valeriote. Decidable unary varieties. Algebra Universalis, 24:1-20, 1987.

[18] M. Valeriote and R. Willard. Discriminating varieties. Accepted by Algebra Universalis, 1991.

[19] Matthew Valeriote. On Decidable Locally Finite Varieties. PhD thesis, University of California, Berkeley, 1986.

[20] R. Willard. Decidable discriminator varieties from unary classes. Transactions of the American Mathematical Society, 336(1):311-333, 1993.

Department of Pure Mathematics, University of Waterloo, Waterloo, Ontario, Canada, N2L 5S7.

Department of Mathematics, University of California, BerkeLEY, CALIFORNIA, USA 94720.

Department of Mathematics and Statistics, McMaster University, Hamilton, Ontario, Canada, L8S 4K1. 